\title{
The Research of AQI Index Changing Regularity Mainly in Tianjin Ziyu Guo
}

\author{
School of Institute of Electrical and Electronic Engineering, North China Electric Power University, \\ Baoding071000, China \\ 540357309@qq.com
}

Keywords:AQI, Guass model, Empirical formula, Temperature

\begin{abstract}
In recent years, the problem of haze is becoming more and more serious in Beijing, Tianjin and Hebei province.Based on the air quality data of January and March 2015, the author has managed to investigate the changing trend of AQI index in Beijing, Tianjin and Hebei by applying a mathematical model. Special attention was paid to find out the relationship between Tianjin and the pollutants in its surrounding area. A mathematical model regarding the factors affect AQI index was built. As the same time, the author also tried to investigate the interrelationship of AQI index among different areas.
\end{abstract}

\section{Introduction}

For the first problem, the author was inspired by the Gauss model and found that the fitting degree of the existing data of the Four order Gauss function was very high. In order for further investigation, the author divide the image of AQI index in the same area in one day into the sum of four Gauss function, and then, put them in the same axis to show for us.By comparing and contrasting, it was found that temperature affected all three Gauss Function. As a result, this research believed the Guass model of the area's AQI is mainly affected by temperature. For the forth uncertain peak, this research paper thought it was due to the uncertain factors like wind power and rain etc. In addition, the effects of its surrounding areas were not negligible.

For the second question, spss software was used to fit the daily AQI index and the AQI index of every hour between different cities. In order to eliminate the third party effect, the author used the Partial correlation analysis and confirm the first question's conclusion of the interrelationships among area's AQI. Through the linear analysis of partial correlation coefficient and distance by using SPSS between the central city and the surrounding cities, we generally found that the relationship between distance and partial correlation coefficient is inversely proportional. If we use $f(r)$ to express the correlation between the cities, we will get $f(r) \propto \frac{1}{r^{\alpha}} \quad(\alpha)$ is the exponent of $r$. Then, this research has done a correlation analysis between an area's various meteorological conditions and AQI by using spss. The author has found that AQI has close relationship between the highest temperature and lowest temperature. Based on this, when excluding the effects of large wind speed and extreme weathers, this research believed a quantitative expression of the distance and temperature can be used to describe the functional relationship of different areas.

\section{Model}

\subsection{The AQI changing regulation in an area based on Gauss model}

Based on the previous modeling preparation, the existing The Gauss diffusion model described the distribution of contaminant concentration in space, the detailed formula is:

$$
C(x, y, z, H)=\frac{Q}{2 \pi v \sigma_{y} \sigma_{z}} \exp \left\{-\frac{1}{2}\left[\frac{y^{2}}{\sigma_{y}^{2}}+\frac{(z-H)^{2}}{\sigma_{z}^{2}}\right]\right\}
$$

In order to obtain the formula of the concentration distribution along $\mathrm{X}$-axis, we made $\mathrm{Z}=0, \mathrm{Y}=0$ and found that 


$$
C(x, 0,0, H)=\frac{Q}{2 \pi v \sigma_{y} \sigma_{z}} \exp \left(-\frac{1}{2} \cdot \frac{H^{2}}{\sigma_{z}^{2}}\right)
$$

above: C----the pollutant concentrations of every point in space(x,y,z)

Q----the strength of source, emissions of pollutants in unit of time

$v$----average speed of wind

$\sigma_{y}, \sigma_{z}$----pollutant diffusion coefficient

Simplyfing the formula, the functions of pollutant concentrationscan changing in space can be obtained

$C(x)=a \cdot e^{-\left(\frac{x-b}{c}\right)^{2}}$

Based on analysis, this pollutant may be due to many aspects. Firstly it was possibly caused by the discharge from an area. In addition, it may also due to the weather conditions like wind or rainfall or surrounding area's effect made AQI fluctuate for certain time. In this research, we call the fluctuation of AQI graph in the area caused by pollutant Gauss impulse.

After doing more research, it was found that we can use Gauss function to best-fit the scatter with many peaks. And a regional AQI curve is usually multimodal, using Gauss function fitting could achieve better result. For the respective functional graph of the AQI scatter points for different timing in one day, we can divide it into a sum of some Gauss functions. By doing so we could match the previous analysis of impact of the area from pollutant. In addition, we could resolve curves of daily AQI to obtain some factors which affect AQI significantly. Through fitting it was found that Four order Gauss fitting could achieve relatively good result. The fitting function is shown below:

$$
y(t)=a_{1} \cdot e^{-\left(\frac{t-b_{1}}{c_{1}}\right)^{2}}+a_{2} \cdot e^{-\left(\frac{t-b_{2}}{c_{2}}\right)^{2}}+a_{3} \cdot e^{-\left(\frac{t-b_{3}}{c_{3}}\right)^{2}}+a_{4} \cdot e^{-\left(\frac{t-b_{4}}{c_{4}}\right)^{2}}
$$

Typical fitting effect is as follows

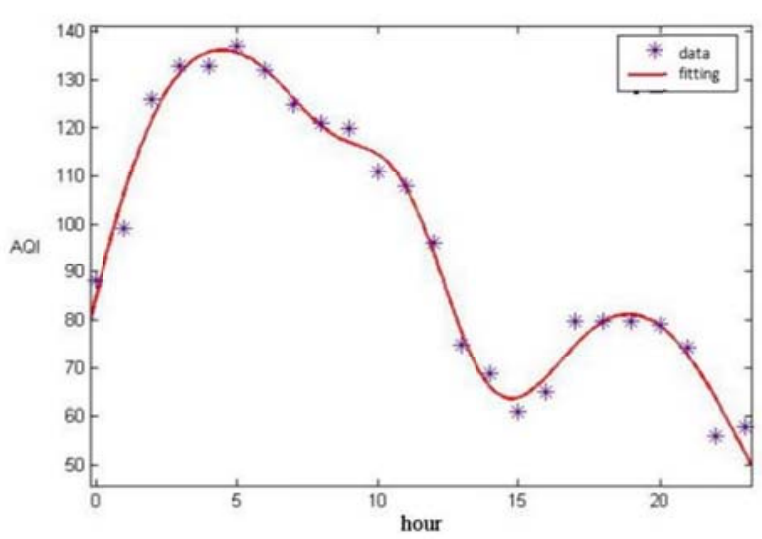

Fig. 1 January 5 th in Tianjin

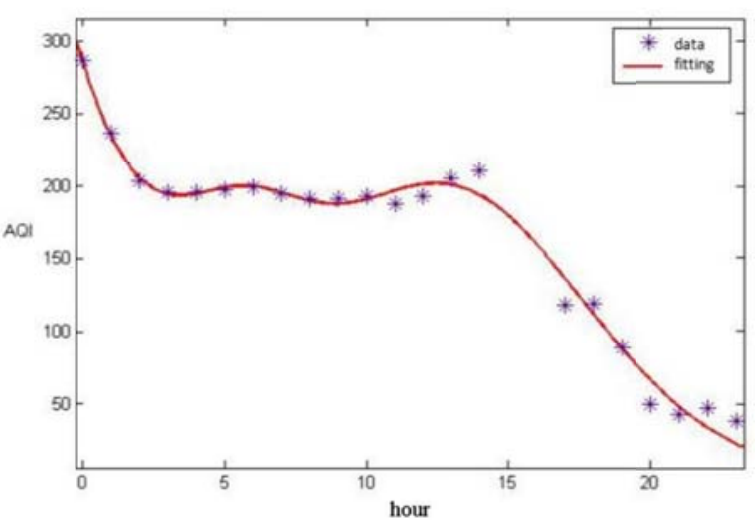

Fig. 2 March 26th in Tianjin

Inspired by this, this paper divided the AQI changing graph of one area in one day into summation of the 4 Gauss function and shown them in the same axis. In order to have a better representativeness, some random days of Shijiazhuang and Tianjin were selected and graph processed. The 4 pictures below were graphs from $3^{\text {rd }}$ March of Shijiazhuang, $24^{\text {th }}$ March, $23^{\text {rd }}$ March and $22^{\text {nd }}$ January of Tianjin respectively.

Based on the four picture above, we could know that an area's AQI changing trend in one day are decided by four factors. Dawn, mid-noon and evening would all produce one Gauss impulse. The positions of the peak value of these 3 Gauss impulse remain the same on the time line. They are at 4'o clock, 12 o'clock and 21 o'clock. Whereas the gauss function caused by other factors(wind, rain etc.) had different peak value position every day. After doing more research, we could know that the effects of dawn, mid-noon and evening were probably due to temperature. The fluctuations of temperature caused the pollutant to Synthesis and decompose and then affect the intensity of pollutant in the air. Besides this, according to Tobler's First Law, the effects among different areas 
are not negligible. Hence, we could know that for the changing of AQI in one day, temperature was the main factor and its effect was also more predictable and stable. The effect form surrounding areas were also comparetivly important. Whereas wind and rains were different every day and their effects were different every day.

Based on the above discussion, using Gauss model to analysis one area's air quality index, this paper thought if we exclude the effect of wind power and extreme weather, the internal environment that impact AQI is probably temperature. However, external environmental factors from surrounding areas may also affect it.

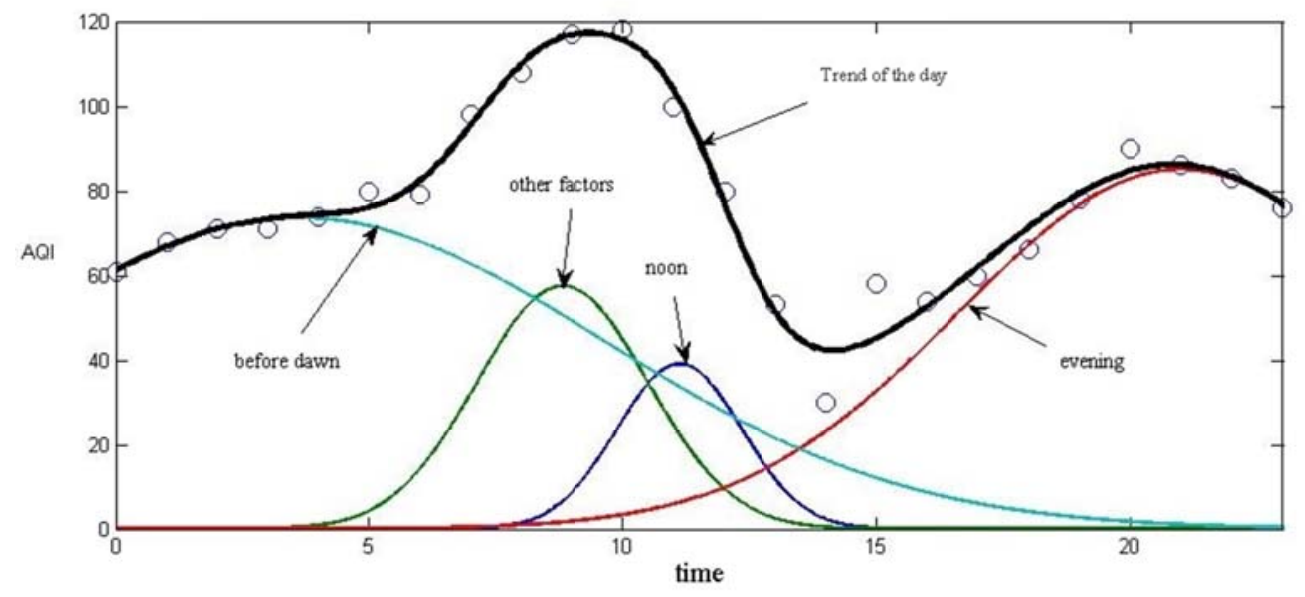

Fig. 3 March $23^{\text {th }}$ in Tianjin

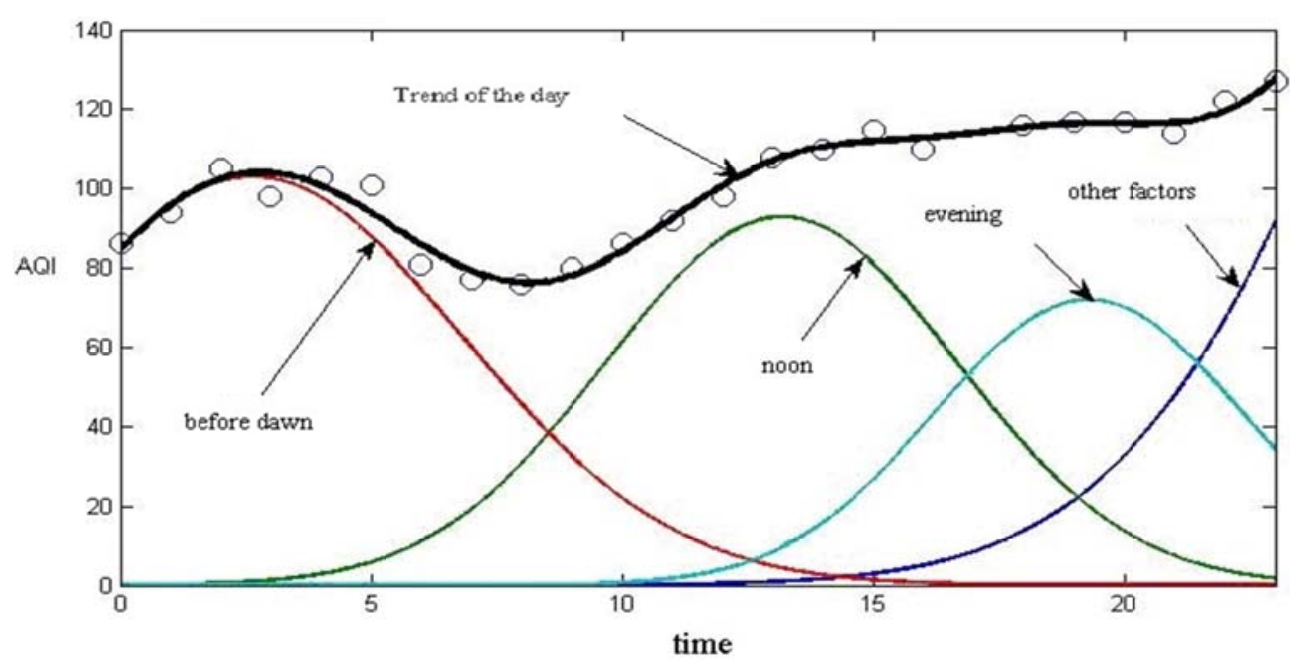

Fig.4 January $22^{\text {nd }}$ in Tianjin

\subsection{The AQI relationships among areas}

Previous by using Gauss fitting, this paper concluded that one area's changing trend were concluded by temperature and surrounding areas. In order to analysis how areas inter-affect each other, this research chose Tianjin and Hebei's cities as the objects. We respectivelyanalyze by SPSS in January, March, as the representative of the relationship between the daily AQI. And randomly chose one hour as unit, cities's AQI relationship and selected 18 cities which has the most significant correlation.

We knew that the pollutant from each city might affect each other or it could be easily affected by third party pollutant. In order to obtain the interrelationship between two cities more accurately, we must exclude the effect from other cities and purely analysis the two cities' net correlation. Hence we use spss to find the 18 cities' partial correlation coefficient respectively. 
Although some areas'AQI change has high correlation, it was affected greatly by pollutants from other areas. We were not hundred percent sure these two cities must inter-affect each other.

In order to exclude the effects of pollutants from other cities, this paper chose 7 pairs of cities' partial correlation coefficient as research objectives.

Table 17 pairs of cities' partial correlation coefficient

\begin{tabular}{|c|c|c|c|c|}
\hline City & Tianjin-Shijiazhuang & Tianjin-Tangshan & Tianjin-Baoding & Tianjin-Langfang \\
\hline Distance(km) & 262.7 & 100.4 & 150.0 & 58.8 \\
\hline $\begin{array}{c}\text { Partial correlation } \\
\text { coefficient }\end{array}$ & 0.229 & 0.256 & 0.266 & 0.383 \\
\hline City & Tianjin-Cangzhou & Tianjin-Chengde & Tianjin-Handan & \\
\hline Distance(km) & 96.1 & 209.9 & 365.0 & \\
\hline $\begin{array}{c}\text { Partial correlation } \\
\text { coefficient }\end{array}$ & 0.261 & 0.234 & 0.190 & \\
\hline
\end{tabular}

After analysis we found that there was an inverse relationship between the correlation and distance. We let $f(r)$ be the correlation among cities, then $f(r) \propto \frac{1}{r^{\alpha}}$, in which $\alpha$ is the exponent of distance $r$. In order to the magnitude of the $\alpha$, this paper use MATLAB curve fitting toolbox, fitting $\alpha$ from 0.2 to 3.We found that when $\alpha$ is 1.5 , the fitting effect is the best. The inter-effect between areas and the distance has an inverse relationship.

When $\mathrm{a}=0.2$ and $\mathrm{a}=1.0$, the fitting effect is as follows:

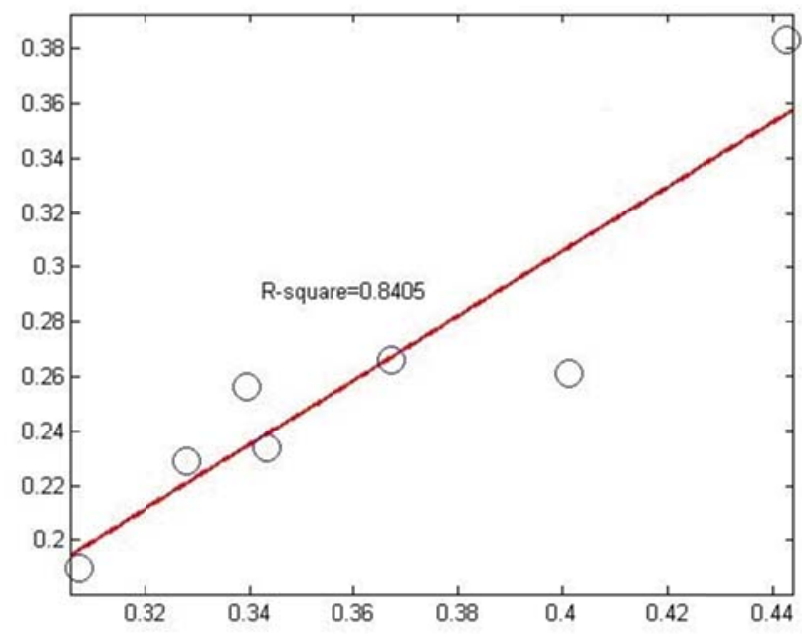

Fig. 5 a $=0.2$ R-square $=0.8406$

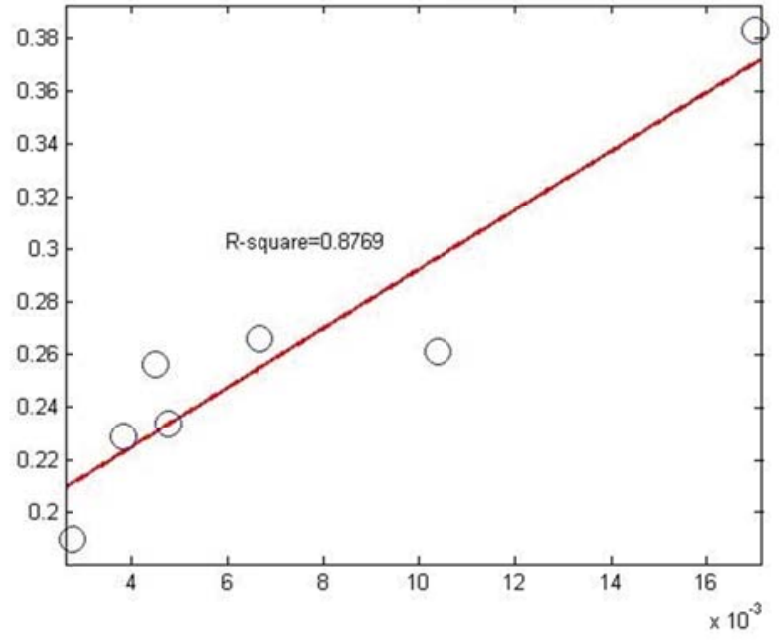

Fig.6.a=1.0 R-square $=0.8769$

When a is different, we can get different Reliability.

Table 2 The different reliability of different ' $a$ '

\begin{tabular}{|c|c|c|c|c|c|}
\hline$\alpha$ & 0.2 & 0.5 & 0.8 & 1.0 & 1.2 \\
\hline$R$-square & 0.8406 & 0.859 & 0.8715 & 0.8769 & 0.8804 \\
\hline$\alpha$ & 1.5 & 1.8 & 2.0 & 2.5 & 3.0 \\
\hline$R$ - square & 0.8824 & 0.8816 & 0.8799 & 0.8733 & 0.8652 \\
\hline
\end{tabular}

After obtaining the correlations for each pair of areas, this paper would investigate how the weather factors affect a area's average AQI. After doing more research, an area's AQI was affected by the hignest temperature, lowest temperature,wind speed, pressure, humidity, rainfall and cloud density. Based on the above Gauss fitting ananlysis, temperature is probably the main factor.

Using the SPSS to ananlysis, Tianjin's January average AQI for hignest temperature, lowest 
temperature and temperature difference were 0.519、0.388、0.267. Tianjin's March average AQI for hignest temperature, lowest temperature and temperature difference were $0.615 、 0.572 、 0.232$. the correlation was quite large. This meant AQI was affected largely by highest temperature and lowest temperature but not temperature difference. This is because the temperature will affect molecules speed and pollutant's diffusion speed. This also match the conclusion drawn from Gauss fitting we discussed above.

The correlation coefficient of Tianjin's January and March average AQI and wind speed were 0.272 and 0.155 respectively. Although correlation coefficient was not very large, it still has some effect.

At the same time, based on the analysis result, the monthly average AQI and other weather's correlation coefficient fluctuate. We could not confirm whether they have clear relationship. However, it was worthy to mention that because Tianjin's January and March has little rainfall hence we could not analyze whether monthly average AQI was affected by rainfall.

Based on the above analysis, daily average AQI was mainly affected by highest temperature and lowest temperature. However, it was weaker relationship with wind speed and other factors. We can assume when the wind power is negligible, daily average AQI was only related to highest temperature and lowest temperature.

$$
\Delta y=\lambda \cdot \sum_{i=1}^{k} \frac{1}{r^{\alpha}} \cdot \Delta y_{i}+F\left(T_{\max }, T_{\min }\right),(\lambda>0)
$$

\section{Summary}

1. the effect among areas were related to distance. Larger distance, smaller effect and smaller distance will lead to large effect.

2. when the distance between areas is extremely small. The inter-effect has a negative correlation with distance. When the distance is very large, the effect will become inversely proportional and even become square inverse relationship. The decreasing trend became flatter.

3. An area was not only affected by its surrounding area, but also affected by its own environment. When the wind power was not extremely strong or it was under extremely bad weather, the main internal environmental factor was temperature. At about 4 am and 10pm a Gauss impulse would be produced and made AQI increase hence decrease the air quality consistently. After doing more research we could know the reason was because the organic pollutant produced by atmosphere chemical reaction will grow at an amazing speed at night. This made the air quality during night very bad. Usually during night, early morning and sunset an inversion layer would be produced easily. This caused the ground temperature to be lower than the temperature in the sky and pollutants in the air would not be able to diffuse. This would match the analysis of effect about morning and night based on Gauss model.Gauss fitting out at noon will generate a Gaussian impulse, and this research was not able to find a reasonable explanation. However, this paper made an assumption that the cumulative effect of pollution source in the morning. The exact reason is waiting for further research.

\section{References}

[1]Jiang Hongbin, Deng Shunxi, Guo Qingni, et al. Based on a modified Gauss model research of diffusion after dangerous gas short time leaked.[J]. Gansu Science, 2013,29 (12): :42-45.

[2]Ding Feng. The explore of Prevention and treatment directions in Beijing,Tianjin and Hebei province.[J]. View, 2014,21:55-56.

[3]Dong He. The application of practical problems about PM 2.5 in the air of Guass smoke and rain model.[N]. The learned journal of eight one Hei Longjiang agriculture university 2014,26(3):69-73. 